\title{
Medial Tibial Stress Syndrome and Female Military Recruits
}

\author{
Rachael Pulsone, Christian Williams \\ Human Services and Rehabilitation, Assumption University, Worcester, USA \\ Email: Rachele81698@gmail.com
}

How to cite this paper: Pulsone, R. and Williams, C. (2022) Medial Tibial Stress Syndrome and Female Military Recruits. Health, 14, 273-280.

https://doi.org/10.4236/health.2022.143021

Received: January 10, 2022

Accepted: February 28, 2022

Published: March 3, 2022

Copyright $\odot 2022$ by author(s) and Scientific Research Publishing Inc. This work is licensed under the Creative Commons Attribution International License (CC BY 4.0).

http://creativecommons.org/licenses/by/4.0/

\begin{abstract}
Upon entering the military recruits are challenged physically, this is exceptionally difficult for women as their anatomy predisposes them to more impact injuries than men. Female military recruits are placed at a higher risk for shin splints, a repetition injury known in the medical field as medial tibial stress syndrome (MTSS). This review paper explores studies about MTSS, its prevalence, and expression in military recruits, particularly as its expression differs across gender. This paper will cover how the external environment of boot camp results in a higher prevalence of MTSS than the general population and how increased occurrence of MTSS in females has been attributed to specific physical attributes. This includes smaller tibial cross-sectional dimensions, greater than average hip range of motion, and differences in lower extremity mechanics during running and other repetitive physical activities. The external environment of boot camp coupled with these intrinsic factors highlight the importance of providing running education for women prior to arrival at boot camp and designing training regiments that account for these physiological differences.
\end{abstract}

\section{Keywords}

Medial Tibial Stress Syndrome, Military, Female, Tibia, Running, Shin Splints, Gender

\section{Introduction}

Medial tibial stress syndrome is an overuse injury defined as pain felt along the lower two thirds of the tibia not due to fracture. MTSS is caused by a high volume of impact activity, over a short period of time. High stress environments like boot camp that involve a recurrent impact exercise have led to a higher incidence of MTSS in recruits compared to the general population [1]. The preva- 
lence of MTSS in the military population is about double the risk of the typical novice athletes [2]. While MTSS should be a concern for all recruits, MTSS is especially concerning for female recruits as their anatomy inherently puts them at an even higher risk for MTSS than their male counterparts [3] [4]. Unfortunately, there are limited studies exploring female military recruits' experience of MTSS. In order to provide female recruits with the best athletic support and education in boot camp, it is vital to understand the etiology of MTSS, and its treatment.

\subsection{History of MTSS}

As early as the 1950s, physicians began to study shin soreness in running athletes. In this time period, shin pain due to repetitive running was believed to be caused by stress fractures to the tibia or shin bone. It was not until 1967 that "Slocum presented a detailed review of the injury, highlighting the fact that shin splints was a specific syndrome with its own clinical symptoms and aetiology" [1]. He argued that shin splints were caused by repetitive running on hard surfaces, like pavement, that lead to excessive use of foot flexors. With the advent of nuclear medicine and bone scanning technologies like the MRI, shin splints, in the medical community, were renamed medial tibial stress syndrome. These new testing abilities ultimately "led to more specific diagnostic criteria for MTSS" [1]. In spite of how far along the medical field has come in terms of advancement and understanding, the field has still yet to agree on a theory of etiology for the disorder.

\subsection{Current Theories of Etiology}

Today there has yet to be a generally agreed consensus of the etiology of medial tibial stress syndrome, currently there are three major theories that divide the field. There are two competing theories that exist in the vast majority of the literature with one having an emphasis in muscular pathologies while the other looks at the influence of cortical bone. A third theory attempts to reconcile the differences of the first two and thus considers the influences that both the musculature and cortical bone play in the role of MTSS.

One recognized theory is that excessive traction of certain leg muscles leads to the pain felt with MTSS. According to Milgrom and colleagues [5], excessive traction of the tibialis posterior and soleus muscles can lead to inflammation of the periosteum, a connective tissue surrounding the tibia. These researchers found there have been no statistically significant findings between which muscle, the tibialis posterior or the soleus, causes inflammation. They further theorize that both have been equally found accountable in contributing to the pain felt during MTSS [5].

A second generally accepted theory is that "medial tibial stress syndrome is a bony overload injury," rather than a muscular one [5]. This theory states the pain felt by MTSS is due to repeated tibial bending or microdamage sustained by 
the cortical bone. In a study performed by Franklyn and colleagues [1], concluded that "MTSS involves cortical bone microfractures". They tested the durability of bovine femora cortical bone when placed under consistently increasing amounts of pressure. Greater amounts of load were associated with greater sustained damage to the cortical bone. This study concluded that under consistent cyclic pressure, "cortical bone fails in both tension and compression" [1].

Finally, the third theory is one that combines both aspects of the traction and boney overload theories [1] [5]. According to Moen and colleagues [4], "The adaptation to loading of the tibia is further challenged by the traction of the soleus and flexor hallucis longus muscles on the periosteum." Whether the traction of the leg muscles or the microdamage to the cortical bone occurs first, is not known. It is known however that the two further exacerbate one another. A smaller cohort study also performed by Franklyn and colleagues [6] found, "female individuals with medial tibial stress syndrome had on average smaller tibial cross-sectional dimensions," compared to men. Because the size of the bone was related to the rate of injury Franklyn and colleagues [1] concluded that medial tibial stress syndrome is not only a soft-tissue injury but also a bony injury. In the past fifty years, it has been concluded that MTSS is not solely due to muscular traction, however how the roles of both muscular and boney theories relate, still requires further research.

\subsection{Diagnosis}

Today when an individual presents with pain in the lower two thirds of the tibia the first course of action is to rule out a stress fracture prior to creating a treatment plan. Stress fractures are on the spectrum of MTSS. In other words, MTSS or a stress reaction can eventually lead to a stress fracture, if left untreated. The two are separate conditions but on the same spectrum. In the field, the current practice for differentiating between two, is that pain and tenderness due to palpation in the affected area less than $5 \mathrm{~cm}$ is indicative of a stress fracture. Meanwhile pain and tenderness due to palpation in an area greater than $5 \mathrm{~cm}$ from the greatest point of pain, is indicative of MTSS [5]. In other words if the pain is sharp and at one single location on the shin, the pain is likely due to fracture. Pain felt more broadly along the shin is usually indicative of MTSS. After physical examination, imaging can also be useful in further ruling out stress fractures [7]. Stress fractures will appear on an x-ray as a strong black line while MTSS will not present on the X-ray. Additionally, "a triple-phase bone scan can show the difference between a stress fracture and medial tibial stress syndrome, while MRI can also exclude tumors/edemas," [8]. Lumps and edemas are atypical of MTSS so if these symptoms persist the clinician can rule out MTSS. In conjunction with palpation and imaging, practitioners can also use the patient's medical history to help aid in diagnosis. Individuals who have experienced MTSS previously, are more prone to experiencing MTSS in the future [3]. It should also be noted that about $1 / 3$ of patients with MTSS will present with a 
comorbid lower leg injury. Overall during evaluation practitioners should use a multifaceted approach in order to ensure the highest level of confidence in their diagnosis. Treatment for a stress fracture and MTSS will differ which is why it is important to be certain of their diagnosis.

\subsection{Recovery}

The most viable treatment for MTSS involves reducing the bone's weight bearing load with rest. Mild MTSS takes about one to two weeks to heal. In the US Naval Academy most individuals who develop MTSS are given an 8-10 day break from running before returning to exercise [9]. If the pain and symptoms do not subside, and more rest is needed, the MTSS may be more severe. Severe MTSS can take anywhere from three to six months to fully recover from [9]. During the recovery period practitioners should consistently score the patients pain along the shin and their physical limitations caused by the pain. This pain scale catalogs the patient's pain at rest, pain while performing activities of daily life, limitations in physical activities, and pain when performing physical activities. Over time as the pain subsides, individuals can gradually return to their prior duties at boot camp.

\section{Methods}

Literary works for this review were selected with search engines and keywords relative to the three major points of this review. The literature included in this review was searched through the use of electronic databases available at; Assumption University Library including the National Center for Biotechnological Information, US Library of Medicine, and PubMed. Searches were also conducted via Google Scholar and additionally, some literary works were located via references found using these search engines.This search included peer reviewed journal articles and scholarly books. Keywords that were used include the history of Medial tibial stress syndrome, risk factors of medial tibial stress syndrome, women and medial tibial stress syndrome, military personnel and medial tibial stress syndrome, differences in men and women in lower extremity functions. A total of 12 articles were used for this review. Articles that were chosen for this review met the requirements of discussing medial tibial stress syndrome in terms of etiology and risk factors, female and military personnel in relation to medial tibial stress syndrome, and information related to the history and theories of medial tibial stress syndrome.

\section{Results}

This literature review found that the external environment of boot camp results in a higher prevalence of MTSS than the general population. Environments such as boot camp and military settings are known for their heavy focus on running related activities and other strenuous physical activities. It was found that high volumes of intense impact exercise can lead to the onset of MTSS over a short 
period of time.

It was also found that females on average are more likely to develop MTSS than their male counterparts [3]. Women on average display physical attributes that put them at a greater risk for MTSS. These physical attributes include smaller tibial cross-sectional dimensions, greater than average hip range of motion, and differences in lower extremity mechanics when running [10]. Therefore, participating in boot camp as a female puts women at an even greater risk for the development of MTSS.

\section{Discussion}

While the etiology of MTSS is multifaceted, and the exact causes are unknown, the progress in the literature reveals certain biological and environmental factors that can put individuals at a higher risk for developing MTSS. It is generally agreed upon that excessive impact exercise, the female sex, smaller than average tibial cross sections, and excessive hip external rotation were associated with a greater incidence of MTSS [3] [10].

According to Milgrom and colleagues [5], MTSS is one of the most common running injuries sustained by military trainees. Boot camp is different from typical athletics in that recruits are engaging in strenuous activities in less than idealized environments. Certain branches of the military stress running more than others because of their designated function in future combat scenarios. Generally the army and marines tend to focus more on running because they are land based troops as compared to the airforce and Navy, whose members are primarily vessel bound. Additionally, more combat oriented branches have the added challenge of training in heavy tactical boots on rough terrain in order to prepare for deployment environments. This can lead to higher impact experienced by the tibia as compared to a typical athlete running on a smooth surface in sneakers. Repeatedly applying a load to the tibia is consistent with the development of microdamage to the cortical bone which can lead to the onset of MTSS [1]. The training environment of the military overall predisposes recruits to higher rates of MTSS than the general population [5]. Recent research has shown that physiological differences between genders also play a role in risk of development for MTSS.

Being of the female gender is a risk factor for developing MTSS [3]. Although the exact cause of why females are predisposed for MTSS is unknown, there are some biologically based theories that may aid this explanation. As stated previously by Franklyn and colleagues [6], women on average have smaller tibial cross-sectional dimensions, compared to men. Her research found that MTSS subjects had "smaller tibial cross-sectional dimensions" than their uninjured individuals. From these statements it can be concluded that the size and strength of the tibia plays a role in the development of MTSS. This would also support the theory that MTSS is partly a boney overload injury. The larger and stronger a bone is, the less likely it is to react under constant stress. This may be one contributing factor as to why women may be more at risk for MTSS. 
Another contributing factor may be that women are known to have greater internal and external ranges of motion that impact running stride. It is known that individuals with greater internal and external ranges of hip motion are placed at a higher risk for MTSS [4] [11]. Greater external hip ranges of motion are associated with a greater knee abduction angle. These two aspects combined lead to a display of different running mechanics than men [11] [12]. A difference is stride is going to lead to a different displacement of the force of impact throughout the leg. In conjunction with a statistically smaller tibia, the displacement of force placed on the tibia when running may explain why females are more susceptible to MTSS than men.

It is known that being in the military puts one at a higher risk for the development of MTSS and it is known that women are at a higher risk for the development of MTSS. The literature suggests that female recruits are therefore put at an even greater disadvantage than their male counterparts. This is evident from Yates and White [9], where they found a significant relationship between gender and MTSS in their study of forty naval recruits. Over the course of boot camp training $53 \%$ of the female recruits developed MTSS while $28 \%$ of their male recruits developed MTSS. While boot camp training can differ slightly between branches, all recruits overall undergo immense amounts of physical stress different from that of civilian athletics. Recruits of all branches must pass some form of a running test and other physical tasks that stress that exert the body. Further research should be done in order to educate future recruits about proper running mechanics and to help develop training plans specific to each branch and their needs. Physical requirements at boot camp should also take into account the gender of the individual inorder to ensure the most optimal conditions for physical and muscular gain throughout training.

\section{Conclusion}

Upon entering the military recruits are put through strenuous high impact training which can be difficult for women as their anatomy predisposes them to more MTSS men. Females generally have smaller tibias, greater range of hip mobility, and overall a broader range of lower extremity mechanics than men. In order to provide female recruits with the best athletic support and education in boot camp, it is vital to understand the etiology of MTSS, and its treatment. The primary aim of this review was to highlight the need for female recruits to receive running education prior to arrival at boot camp as well as recommend stringent training programs in boot camp that take into account female's strengths and deficits. It is recommended that most recruits start training for a minimum of four to six months to prior arrival at boot camp. A proper running program if followed for four to six months prior to the arrival at boot camp can help prevent injury mid training. MTSS is an overuse injury caused by high impact activities. If recruits take the time to gradually load and strengthen their lower extremities they can decrease their risk of injury. The tibia can only withstand so 
much force over a short period of time, this is why it is recommended that recruits should prepare to be as physically fit as they can, prior to arrival at boot camp. Longitudinal studies would be required to both determine the benefits of providing running education for women prior to arrival at boot camp and to guide training regiments that may be more effective with the female population.

\section{Acknowledgements}

I would like to thank Dr. Williams and the Human Services and Rehabilitation department at Assumption University for granting me the time and resources to put this literature review together.

\section{Conflicts of Interest}

The authors do not have any conflict of interest.

\section{References}

[1] Franklyn, M. and Oakes, B. (2015) Aetiology and Mechanisms of Injury in Medial Tibial Stress Syndrome: Current and Future Developments. World Journal of Orthopedics, 6, 577-589. https://www.wjgnet.com/2218-5836/full/v6/i8/577.htm https://doi.org/10.5312/wjo.v6.i8.577

[2] Campbell, J. (2019) An Exploratory Study of Military Management Practices, Physical Activity, and the Prevalence of Shin Splints in ROTC Cadets. Electronic Theses and Dissertations Paper 3383. https://dc.etsu.edu/etd/3383

[3] Bliekendaal, S., Moen, M., Fokker, Y., Stubbe, J.H., Twisk, J. and Verhagen, E. (2018) Incidence and Risk Factors of Medial Tibial Stress Syndrome: A Prospective Study in Physical Education Teacher Education Students. BMJ Open Sport \& Exercise Medicine, 4, e000421. https://doi.org/10.1136/bmjsem-2018-000421

[4] Moen, M.H., Tol, J.L., Weir, A., Steunebrink, M. and De Winter, T.C. (2012) Medial Tibial Stress Syndrome: A Critical Review. Sports Medicine, 39, 523-546.

https://doi.org/10.2165/00007256-200939070-00002

[5] Milgrom, C., Zloczower, E., Fleischmann, C., Spitzer, E., Landau, R., Bader, T. and Finestone, A.S. (2020) Medial Tibial Stress Fracture Diagnosis and Treatment Guidelines. Journal of Science and Medicine in Sport, 24, 526-530. https://doi.org/10.1016/j.jsams.2020.11.015

[6] Franklyn, M., Oakes, B., Field, B., Wells, P. and Morgan, D. (2008) Section Modulus Is the Optimum Geometric Predictor for Stress Fractures and Medial Tibial Stress Syndrome in Both Male and Female Athletes. The American Journal of Sports Medicine, 36, 1179-1189. https://doi.org/10.1177/0363546508314408

[7] Amin, I. and Moroz, A. (2017) Medial Tibial Stress Syndrome (Shin Splints). Musculoskeletal Sports and Spine Disorders. Springer, Cham. https://doi.org/10.1007/978-3-319-50512-1 63

[8] Physiopedia Contributors (2019) Shin-Splints. Physiopedia. https://www.physio-pedia.com/index.php?title=Shin-splints\&oldid=221901

[9] Yates, B. and White, S. (2004) The Incidence and Risk Factors in the Development of Medial Tibial Stress Syndrome among Naval Recruits. The American Journal of Sports Medicine, 32, 772-780. https://doi.org/10.1177/0095399703258776

[10] Winkelmann, Z.K., Anderson, D., Games, K.E. and Eberman, L.E. (2016) Risk Fac- 
tors for Medial Tibial Stress Syndrome in Active Individuals: An Evidence-Based Review. Journal of Athletic Training, 51, 1049-1052.

https://doi.org/10.4085/1062-6050-51.12.13

[11] Czuppon, S., Prather, H., Hunt, D.M., Steger-May, K., Bloom, N.J., Clohisy, J.C., Larsen, R. and Harris-Hayes, M. (2017) Gender-Dependent Differences in Hip Range of Motion and Impingement Testing in Asymptomatic College Freshman Athletes. $P M \& R$ : The Journal of Injury, Function, and Rehabilitation, 9, 660-667.

https://doi.org/10.1016/j.pmrj.2016.10.022

[12] Ferber, R., Davis, I.M. and Williams 3rd, D.S. (2003) Gender Differences in Lower Extremity Mechanics during Running. Clinical Biomechanics (Bristol, Avon), 18, 350357. https://doi.org/10.1016/S0268-0033(03)00025-1 